\title{
Build high-resolution global climate models
}

\section{International supercomputing centres dedicated to climate prediction are needed to reduce uncertainties in global warming, says Tim Palmer.}

\section{$\mathrm{T}$} The drive to decarbonize the global economy is usually justified by appealing to the precautionary principle: reducing emissions is warranted because the risk of doing nothing is unacceptably high. By emphasizing the idea of risk, this framing recognizes uncertainty in the magnitude and timing of global warming.

This uncertainty is substantial. If warming occurs at the upper end of the range projected in the Intergovernmental Panel on Climate Change (IPCC) Fifth Assessment Report ${ }^{1}$, then unmitigated climate change will probably prove disastrous worldwide, and rapid global decarbonization is paramount. If warming occurs at the lower end of this range, then decarbonization could proceed more slowly and some societies' resources may be better focused on local adaptation measures.

Reducing these uncertainties substantially will take a new generation of global climate simulators capable of resolving finer details, including cloud systems and ocean eddies. The technical challenges will be great, requiring dedicated supercomputers faster than the best today. Greater international collaboration will be needed to pool skills and funds.

Against the cost of mitigating climate change - conceivably trillions of dollars - investing, say, one quarter of the cost of the Large Hadron Collider (whose annual budget is just under US $\$ 1$ billion) to reduce uncertainty in climate-change projections is surely warranted. Such an investment will also improve regional estimates of climate change — needed for adaptation strategies - and our ability to forecast extreme weather.

\section{GRAND CHALLENGES}

The greatest uncertainty in climate projections is the role of the water cycle - cloud formation in particular - in amplifying or damping the warming effect of $\mathrm{CO}_{2}$ in the atmosphere ${ }^{2}$. Clouds are influenced strongly by two types of circulation in the atmosphere: mid-latitude, low-pressure weather systems that transport heat from the tropics to the poles; and convection, which conveys heat and moisture vertically.

Global climate simulators calculate the evolution of variables such as temperature, humidity, wind and ocean currents over a grid of cells. The horizontal size of cells in current global climate models is roughly 100 kilometres. This resolution is fine enough to simulate mid-latitude weather systems, which stretch for thousands of kilometres. But it is insufficiently fine to describe convective cloud systems that rarely extend beyond a few tens of kilometres.

Simplified formulae known as 'parameterizations' are used to approximate the average effects of convective clouds or other small-scale processes within a cell. These approximations are the main source of errors and uncertainties in climate 
simulations ${ }^{3}$. As such, many of the parameters used in these formulae are impossible to determine precisely from observations of the real world. This matters, because simulations of climate change are very sensitive to some of the parameters associated with these approximate representations of convective cloud systems ${ }^{4}$.

Decreasing the size of grid cells to 1 kilometre or less would allow major convective cloud systems to be resolved. It would also allow crucial components of the oceans to be modelled more directly. For example, ocean eddies, which are important for maintaining the strength of larger-scale currents such as the Gulf Stream and the Antarctic Circumpolar Current, would be resolved.

The goal of creating a global simulator with kilometre resolution was mooted at a climate-modelling summit in $2009^{5}$. But no institute has had the resources to pursue it. up to the task. Modelling efforts have instead focused on developing better representations of ice sheets and biological and chemical processes (needed, for example, to represent the carbon cycle) as well as quantifying climate uncertainties by running simulators multiple times with a range of parameter values.

Running a climate simulator with 1-kilometre cells over a timescale of a century will require 'exascale' computers capable of handling more than $10^{18}$ calculations per second. Such computers should become available within the present decade, but may not become affordable for individual institutes for another decade or more.

\section{CLIMATE FACILITIES}

The number of low-resolution climate simulators has grown: 22 global models contributed to the IPCC Fourth Assessment Report in 2007; 59 to the Fifth Assessment Report in 2014. European climate institutes alone And, in any case, current computers are not

contributed 19 different climate model integrations to the Fifth Assessment database (go. nature.com/3gu8co). Meanwhile, systematic biases and errors in climate models have been only modestly reduced in the past ten years ${ }^{6}$.

It is time to establish a small number of international climate-prediction facilities ${ }^{5,7}$, in which climate institutes, weather-forecast centres and academic departments can combine resources and talents to create the first cloud-resolved global climate simulators within a decade. Focusing on fewer simulators, perhaps one per continent, would avoid duplication and concentrate the large number of individually poorly resourced efforts, yet maintain a competitive environment to encourage scientific innovation.

The success of the European Centre for Medium-Range Weather Forecasts, an intergovernmental effort, is a good example. The centre was set up in the 1970s to produce weather forecasts up to ten days ahead using a global weather model. From the beginning, its forecasts have been the envy of the world. Funding from the centre's 34 member states enables human talent to be drawn from across Europe with jointly funded supercomputing infrastructure.

This concept now needs to be applied to climate prediction. A budget of a few hundred million euros a year from European governments, the European Union and perhaps the private sector could support such a centre in Europe. A multi-agency initiative might establish a facility in North America. Leading countries in climate research such as China, India, Japan and Korea might jointly fund a facility in Asia.

Computational challenges will have to be overcome. For example, for software to run efficiently on exascale computers comprising a million or more independent processing elements, only essential information can be passed between processors, and from

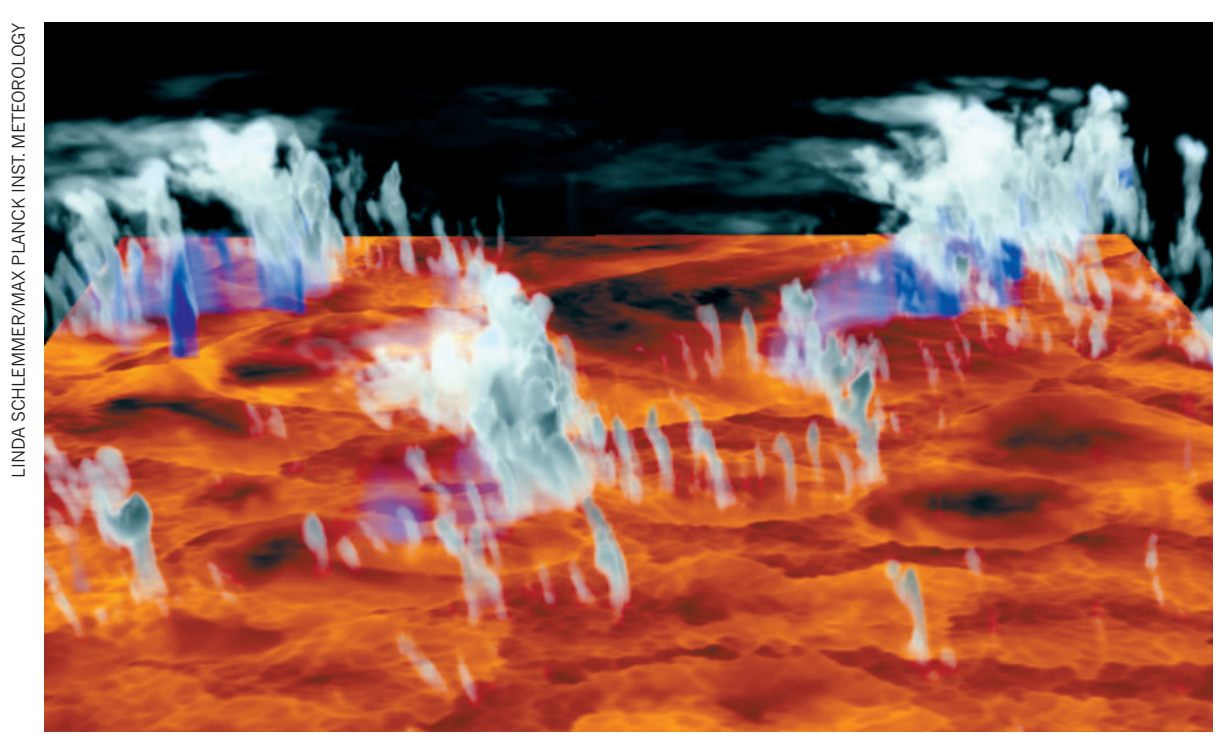

Simulation of convective cloud systems in a limited-area high-resolution climate model.

processor to memory. Climate and computer scientists will need to assess the physical information content in the millions of climatic variables described ${ }^{8,9}$. This will also be relevant in deciding at what level of detail the plentiful model data must be archived. Computer hardware will need to evolve to allow the efficient computation, transmission and storage of model variables with a range of numerical precision.

Even with 1-kilometre cells, unresolved cloud processes such as turbulence and the effects of droplets and ice crystals will have to be parameterized (using stochastic modelling to represent uncertainty in these parameterizations $\left.{ }^{9}\right)$. How, therefore, can one be certain that global-warming uncertainty can be reduced? The answer lies in the use of 'data assimilation' software - computationally demanding optimization algorithms that use meteorological observations to create accurate initial conditions for weather forecasts. Such software will allow detailed comparisons between cloud-scale variables in the highresolution climate models and corresponding observations of real clouds, thus reducing uncertainty and error in the climate models ${ }^{10}$.

High-resolution climate simulations will have many benefits beyond guiding mitigation policy. They will help regional adaptation, improve forecasts of extreme weather, minimize the unforeseen consequences of climate geoengineering, and be key to attributing current weather events to climate change.

High-energy physicists and astronomers have long appreciated that international cooperation is crucial for realizing the infrastructure they need to do cutting-edge science. It is time to recognize that climate prediction is 'big science' of a similar league.

Tim Palmer is a Royal Society research professor of climate physics and co-director of the Oxford Martin Programme on Modelling and Predicting Climate at the University of Oxford, UK.

e-mail:t.n.palmer@atm.ox.ac.uk

1. Stocker, T. F. et al. (eds) Climate Change 2013: The Physical Science Basis. Contribution of Working Group I to the Fifth Assessment Report of the Intergovernmental Panel on Climate Change (Cambridge Univ. Press, 2013).

2. Stevens, B. \& Bony, S. Science 340, 1053-1054 (2013).

3. Jakob, C. Bull. Am. Meteorol. Soc. 91, 869-875 (2010).

4. Sherwood, S. C., Bony, S. \& Dufresne, J.-L. Nature 505, 37-42 (2014).

5. Shukla, J. et al. Bull. Am. Meteorol. Soc. 91, 1407-1412 (2010).

6. Rauser, F., Gleckler, P. \& Marotzke, J. Bull. Am. Meteorol. Soc. (in the press).

7. Palmer, T. N. Physics World 24, 14-15 (2011)

8. Palmer, T. N., Düben P. \& McNamara, H. Phil. Trans. R. Soc. A 372, 20140118 (2014).

9. Palmer, T. N. Q. J. R. Meteorol. Soc. 138, 841-861 (2012).

10.Rodwell, M. J. \& Palmer, T. N. Q. J. R. Meteorol. Soc. 133, 129-146 (2007). 